\title{
Direct Lattice Parameter Measurements Using HAADF-STEM
}

\author{
Adedapo A. Oni ${ }^{1}$, Xiahan Sang ${ }^{1}$, Aakash Kumar ${ }^{2}$, Selva V. Raju ${ }^{3}$, Srikant Srinivasan ${ }^{4}$, Susan Sinnott ${ }^{2}$, \\ Surendra Saxena ${ }^{3}$, Krishna Rajan ${ }^{4}$, James M. LeBeau ${ }^{1}$ \\ 1. North Carolina State University, Materials Science and Engineering, Raleigh, USA. \\ 2. University of Florida, Materials Science and Engineering, Gainsville, USA. \\ 3. Florida International University, Mechanical and Materials Engineering, Miami, USA. \\ 4. Iowa State University, Materials Science and Engineering, Ames, USA.
}

Lattice strain is generated in crystal structures as a result of atomic size differences between host atom and solute elements during substitutional alloying. Extensive work has been performed to study lattice parameter variation with alloying elements, primarily using diffraction methods. The global information provided by reciprocal space analysis, however, limits access to local structural details. In contrast, atomic resolution STEM enables direct imaging of the crystal structure, but drift distortion currently limits capabilities to measure lattice parameters. This is particularly relevant for Ni-based superalloys as the microstructure consists of cuboidal intermetallic $\gamma^{\prime}$ phase precipitate $\left(\mathrm{L}_{2}\right.$ structure) within a $\gamma$ phase matrix (FCC structure). As the coherent $\gamma / \gamma$ ' interface is responsible for limiting dislocation motion [1], direct measurement of lattice parameters and strain provides critical information to further next generation alloy design.

In this presentation, we will report direct lattice parameter measurements in annealed single-crystal Ni-based superalloys using a probe-corrected scanning transmission electron microscope (FEI Titan 60-300 S/TEM). We will show that picometer precision and accuracy are enabled by the newly developed RevSTEM technique [2]. Peak locations are determined using a normalized cross correlation approach and 2D Gaussian peak fitting [2] to enable the measurement of atom column separations. RevSTEM-processed HAADF-STEM images for pure $\mathrm{Ni}_{3} \mathrm{Al}, \gamma^{\prime}$ phase $\mathrm{NiAlCr}$ and $\gamma$ phase NiAlCr in the $<100>$ projection are shown in Figure 1. A histogram of the third and fourth nearest-neighbor distances calculated from the HAADF-STEM images is presented in Figure 2. The lattice parameter measurements using RevSTEM images acquired from each of the three phases are consistent with the expected trend $a\left(\mathrm{Ni}_{3} \mathrm{Al}\right)>a\left(\mathrm{NiAlCr} \gamma^{\prime}\right)>a(\mathrm{NiAlCr} \gamma)$ [3-4]. Extending the approach, we will demonstrate the power of the technique to accurately measure lattice parameter and strain across the $\gamma /$ $\gamma$ ' interface. Overall, these results open a new realm of atomic scale structure analysis that was previously just beyond our reach due to drift [2].

\section{References:}

[1] RC Reed in "The superalloys: Fundamentals and Applications", (Cambridge University Press, Cambridge).

[2] X Sang and JM LeBeau, Ultramicroscopy 138 (2014) 28-35.

[3] Q Wu and S Li, Computational Materials Science 53 (2012) 436-443.

[4] Y Mishima et al., Acta Metall. 33 (1985) 1161-1169.

[5] The authors acknowledge support from the Air Force Office of Scientific Research (Grant No. FA9550-12-1-0456). 


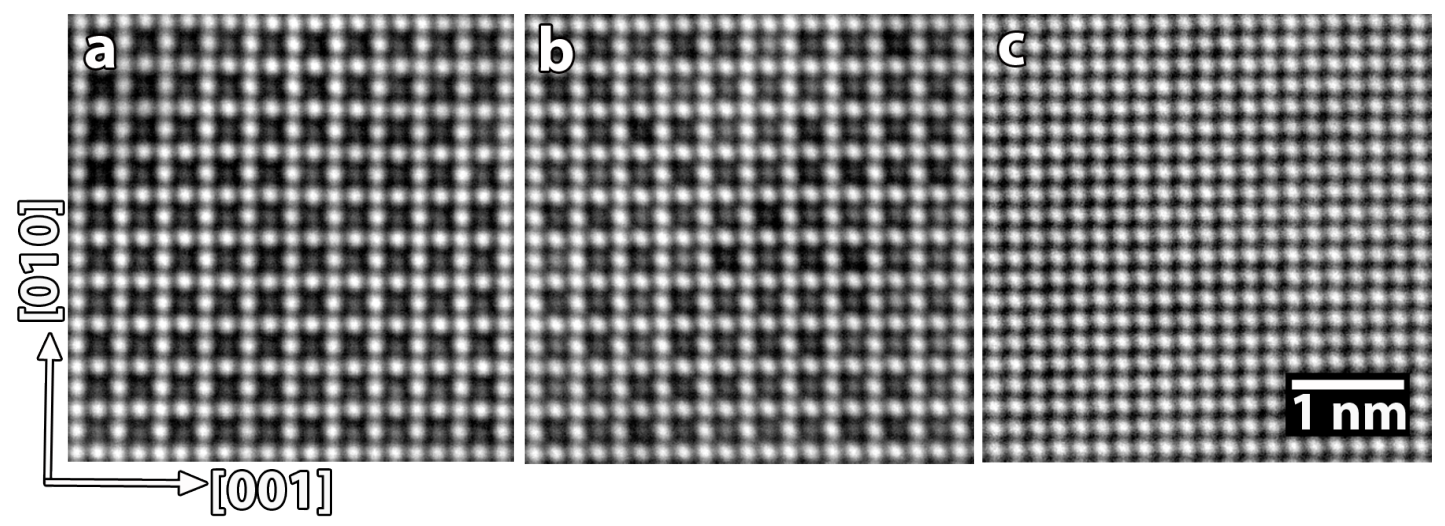

Figure 1. HAADF-STEM images of (a) pure $\mathrm{Ni}_{3} \mathrm{Al}$ (b) $\gamma^{\prime}$ NiAlCr and (c) $\gamma \mathrm{NiAlCr}$ along the $<100>$ zone.

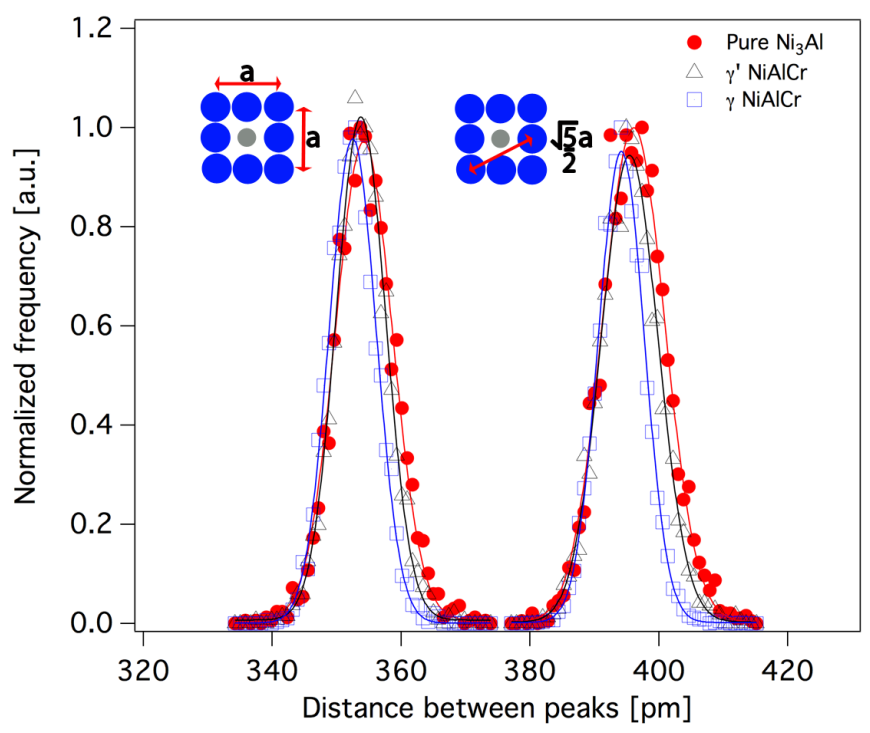

Figure 2. Corresponding histograms of the third and fourth nearest-neighbor distances calculated from HAADF-STEM images above. The mean lattice parameters are consistent with the expected trend $\mathrm{a}\left(\mathrm{Ni}_{3} \mathrm{Al}\right)>a\left(\mathrm{NiAlCr} \gamma^{\prime}\right)>a(\mathrm{NiAlCr} \gamma)[3-4]$. 